\title{
Sharp inequalities for the harmonic numbers
}

\section{CHAO-PING CHEN}

\section{ABSTRACT.}

Let $H_{n}$ be the $n$th harmonic number, and let $\gamma$ be the Euler-Mascheroni constant. We prove that for all integers $n \geq 1$, the double-inequality

$$
\alpha-\ln \left(e^{2 \sqrt{2} /(4 n+2+\sqrt{2})}-1\right)<H_{n} \leq \beta-\ln \left(e^{2 \sqrt{2} /(4 n+2+\sqrt{2})}-1\right)
$$

holds with the best possible constants

$$
\alpha=\gamma-\frac{1}{2} \ln 2=0.2306 \ldots \text { and } \beta=1+\ln \left(e^{2 \sqrt{2} /(6+\sqrt{2})}-1\right)=0.2331 \ldots .
$$

We also establish inequality for the Euler-Mascheroni constant

$$
\frac{\sqrt{2}}{96 n^{3}}-\frac{13+18 \sqrt{2}}{1152 n^{4}}<\mu_{n}-\gamma<\frac{\sqrt{2}}{96 n^{3}}
$$

where

$$
\mu_{n}=H_{n}+\ln \left(e^{2 \sqrt{2} /(4 n+2+\sqrt{2})}-1\right)+\frac{1}{2} \ln 2 .
$$

HenAN POLYTECHNiC UNIVERSITY

SCHOOL OF MATHEMATICS AND INFORMATICS

Jiaozuo City 454003, Henan Province, People's Republic of China

E-mail address: chenchaopingesohu.com

Received: 31.01.2011; In revised form: 24.11.2011; Accepted: 23.04.2012

2010 Mathematics Subject Classification. 11B83, 11Y60, 26D15.

Key words and phrases. Harmonic numbers, Euler-Mascheroni constant, inequalities. 